\title{
PANORAMA DE LOS VERBOS DEPONENTES EN EL LATIN BAJOMEDIEVAL
}

\author{
por Estrella PEREZ RODRIGUEZ (Universidad de Valladolid)
}

La categoría de los deponentes, a la que pertenecía un número nada despreciable de verbos, supuso desde un principio una anomalía dentro del sistema verbal latino. En el transcurso de la historia de esta lengua dichos verbos sufrieron fluctuaciones, concurrencias, modificaciones, que, sin embargo, no llegaron a perjudicar la estabilidad de que gozaba este grupo de verbos. Dentro de esas variaciones a las que se ven sometidos no son precisamente las más numerosas, como se creyó durante mucho tiempo, aquéllas por las que los verbos deponentes dejaban de ser tales para pasar a activos. Por el contrario, según ha demostrado P. Flobert en su intenso y completísimo estudio al respecto ', es más frecuente que los verbos activos se «deponenticen». Todo ello hace que incluso en una misma época se pueda encontrar un verbo utilizado indistintamente como deponente o como activo/pasivo. No obstante, con el paso del tiempo esa lenta erosión conduce a la situación ya observable a mediados del s. VIII, donde el deponente -al menos en el infectumparece haberse convertido en «una pura variante morfológica del activo» 2.

El mencionado estudio de Flobert describe la situación de los verbos deponentes en su utilización práctica hasta la época de Carlomagno, pero iqué ocurre con éstos a partir de entonces, en el latín medieval? Eso es precisamente lo que nos proponemos tratar aquí, pero sometiéndonos a ciertas limitaciones. En primer lugar, nos serviremos, no del uso de cada verbo en los textos, sino de la propia opinión de los gramáticos medievales. Hemos escogido, pues, para realizar nuestro trabajo dos obras teóricas de naturaleza lexicográfica, pertenecientes ambas al s. XIII, pero localizada cada una en uno de sus extremos. La más temprana, el Verbiginale ${ }^{3}$, se puede fechar a comienzos de dicho siglo y consiste en un estudio individual en verso y por orden alfabético de los verbos latinos; nos serviremos igualmente del extenso comentario que la acompaña, también del s. XIII. La segunda es un léxico compuesto en 1286 por el italiano Iohannes Balbus bajo el título de Catholi-

(1) Les verbes déponents latins des origines à Charlemagne, Paris, 1975.

(2) P. FLOBERT, o.c., p. 571.

(3) El estudio y edición crítica de esta obra prácticamente desconocida lo hemos realizado en nuestra Tesis Doctoral titulada: El «Verbiginale» de «Petrus Palentinus», una gramática medieval en verso. Edición crítica y estudio. 
con. Ambas tienen una fuente en común del siglo precedente, las Derivationes de Hugutio de Pisa, unida a su vez con toda una tradición anterior, razón por la que las dos obras pueden ser buena muestra de una situación más general que la del siglo al que se adscriben ${ }^{4}$.

Verbiginale y Catholicon, que, aunque extensos, no son -como es fácil imaginar- exhaustivos, suelen indicar explícita y puntualmente el género de cada uno de los verbos tratados. A ello los mueve su finalidad docente. Según las enseñanzas gramaticales vigentes en la época, heredadas de los gramáticos del Bajo Imperio, existían cinco géneros verbales diferentes: activo, pasivo, neutro, común y deponente, en ese orden. Puesto que se consideraba este accidente verbal como la conjunción de desinencia y significado, se conserva la definición tradicional de deponente como illud quod in passiva literatura retinet tantum significationem actiuam ${ }^{5}$. Petrus Helias es mucho más explícito y, después de hacer hincapié en que la característica principal de este tipo de verbos es expresar, a diferencia del común, una sola significación, ya sea ésta activa o pasiva, ${ }^{6}$ especifica las cuatro diferentes significaciones que, según él, puede tener un verbo deponente 7 , es decir, cualquiera mientras sea única.

Tras observar el subgrupo de verbos que reciben de esta obra la calificación de deponentes, podemos dividirlos en cuatro tipos distintos, atendiendo únicamente a los datos que sobre ellos nos proporcionan ambas gramáticas.

1.-Verbos que, según ellas, son siempre y exclusivamente deponentes y no se reconocen para ellos alternancias o fluctuaciones con otro género. Es la clase más amplia con diferencia sobre el resto.

2.-Aquellos verbos que junto a su forma deponente pueden tener una segunda activa o neutra (o ambas) sin que la utilización de una $u$ otra suponga un cambio de significado o una construcción diferente. Cuando el verbo presenta los tres géneros, existen diferencias del tipo señalado entre activo y neutro, mientras que el deponente suele ser libremente intercambiable con cualquiera de ellos o con ambos. Si sobre el verbo en cuestión se forman compuestos con la ayuda de preverbios, éstos se comportan de forma idéntica a su simple.

(4) Las opiniones del Verbiginale y su comentario han sido tomadas de la mencionada edición que incluye a ambos. Las del Catholicon lo han sido de la edición de Mainz, 1460 (repr. 1971). Puesto que ambas obras siguen el orden alfabético, permitasenos para aliviar el material aquí presentado obviar la indicación de su emplazamiento en cada una.

(5) IOHANNES BALBUS, Catholicon, Mainz, 1460, gramm. (s. p.).

(6) Dicatur deponens quia alteram deponit significationem secundum quod uidebitur deponens dici respectu communis. Bene autem dixit unam et non alteram determinute quia sunt quedam que solam actionem significant ut loquo, quedam passionem ut nascor Commentum super Priscianum Maiorem, París, Biblioteca del Arsenal, ms. 711, f. $63 r$ ).

(7) Sunt quedam enim deponentium que actiuam habent significationem... Subiunge uero postea secundam... que est passio illata ab alio ut pacior a te, mereor a te... Tercia deponentium significatio est absoluta actio uel passio intrinseca... Quarta... est passio nata intrinsecus sed transiens ut obliuiscor... (Commentum..., ff. $64 \mathrm{r}-64 \mathrm{v}$ ).

(8) Todos estos datos y los referentes a los demás verbos corresponden a la teoria transmitida por las dos obras estudiadas. 
-Adaquor. es deponente, pero se puede encontrar también como activo y lo ilustran con una cita: unde in Theodolo: "enadaquare gregem simul et leuare calorem nostra uenit fronesis». Rige acusativo.

-Ancillor: cuando significa "administrare», es activo y se construye con acusativo y dativo. Cuando significa «servire», es neutro y se construye únicamente con dativo. Con este mismo significado se puede encontrar también como deponente.

-Aspernor compuesto de ad y sperno, pertenece a la primera conjugación y se construye con acusativo. Puede aparecer como activo o como deponente.

-Cauillor: con el mismo significado de «decipere», se puede encontrar como activo o como deponente. Rige acusativo.

-Exsecror: compuesto de ex y sacro. Es activo, pero a veces puede aparecer como deponente. El Catholicon precisa: frequenter inuenitur execror.

-Feneror. es neutro o deponente con el mismo significado: «mutuare uel mutuari, .i. dare ad usuram uel accipere». De igual forma se comportan sus compuestos confeneror, refeneror, defeneror. Cuando este verbo significa «coniungere» o «ligare», es activo. Precisamente es éste un deponente junto al que desde muy pronto aparece una forma activa que acabará predominando sobre aquélla 9 .

-Ferior: verbo absoluto, derivado de feria que puede hallarse como neutro o deponente. Se especifica que esa segunda forma es la que más está en uso.

También pertenecen a este grupo gesticulor (deponente o neutro); malignor (activo o deponente); medicor (deponente o activo); nictor (neutro o deponente junto con sus compuestos innictor y adnictor); odoror (activo o deponente, para ilustrar este último se pone un ejemplo de la Vulgata); ringor (neutro o deponente al igual que su compuesto arringor); los compuestos de spico : conspicor, despicor, exspicor; suspicor (compuesto de susum y specio, puede ser activo o deponente); tribulor (activo o deponente), y lo mismo uelificor, uociferor, etc.

3.-Verbos que se pueden encontrar también bajo dos géneros diferentes pero a cada uno de ellos le corresponde un significado y una construcción particular. Ambas obras los suelen considerar como verbos distintos.

- Mutuo -as / mutuor -aris: el primero es activo, se construye con acusativo y dativo y significa «dare», deriva de mutuum y se compone commutuo e immutuo. El segundo es deponente, se construye con acusativo y ablativo y significa «accipere», deriva también de mutuum y se compone commutuor e immutuor.

-Tristo - as / tristor - aris: el primero es activo, rige acusativo y significa «tristem facere, dolere». El segundo es deponente absoluto y significa «dolere, tristem essen. El mismo comportamiento sigue el compuesto de ambos contristo / contristor.

(9) P. FLOBERT, o.c., pp. 96-7. 
4.-Verbos que en un pasado sin delimitar se utilizaban bajo un género distinto al de esa época.

-Deponentes en la antigüedad: munero, palpo (antiqui dicebant palporparis deponens pro "adulari», CATHOL. s.v.); conuertor y praeuertor eran deponentes, pero ahora deben ser considerados meramente pasivos.

-Deponentes en la actualidad, pero no antiguamente: calumpnior (realmente no se encuentra como activo hasta aproximadamente el s. III d.C. 10); comitor; complector que según el Catholicon antiguamente era común; hospitor, también común en la antigüedad; de ludificor se dice que era activo, pero al mismo tiempo se da una cita de Plauto en la que aparece como deponente; modulor (activo antiguamente, aunque realmente no aparece como tal hasta los ss. IV-V d. C. II); patior y sus compuestos; predor y sus compuestos; tutor $y$ contutor; uador (común entre los antiguos), etc.

A juzgar por todo esto, parece claro que en el latín medieval se continúa teniendo conciencia de la clase de los verbos deponentes y de las particularidades que los caracterizan y éstas se siguen respetando o, al menos, se intentan respetar. La situación que reflejan Verbigiale y Catholicon no es muy diferente, en líneas generales, a la de cualquier periodo de los estudiados por Flobert. Tampoco aquí están los deponentes libres de fluctuaciones o vacilaciones, aunque pueda sorprender el predominio de aquéllos a los que se cataloga como tales sin apuntar mayores problemas. Sin embargo, esto no resulta en absoluto extraño, si tenemos en cuenta el terreno en el que nos estamos moviendo: obras teóricas de adoctrinamiento, cuya misión es fijar esquemas y establecer certezas que ofrecer a la memoria. Las activaciones, pasivaciones o deponentizaciones de que habla Flobert se presentan en ellas en la mayoría de los casos «institucionalizadas», consagradas por la norma. Pero esta norma no siempre es unitaria: p.e. para el Verbiginale es assentior deponente, según el Catholicon se le puede encontrar también como activo; auguror es deponente para aquél, pero para éste también puede ser neutro; el Verbiginale considera deponente a geniculor, mientras que el otro lo cataloga como activo o deponente; por el contrario latinizor puede aparecer como neutro o deponente según aquél y sólo como neutro según éste; el Catholicon añade a naufragor un doblete activo, que desconoce el Verbiginale; exactamente al contrario se comportan en el caso de uociferor; etc. En la misma linea está el hecho de que los compuestos sigan manteniendo también en el latín medieval habitualmente el género de sus simples, salvo excepciones ya antiguas (auertor, reuertor, conspicor,...), lo mismo sucede con los derivados con respecto al género de sus primitivos (sciscitor y scitor suponen una ruptura de esta regla especialmente resaltada aqui, aunque lo venía siendo ya desde Prisciano). El latín medieval como la cultura de esa época son herederos de lo anterior y lo asumen como propio dentro de sus limitaciones y falta de juicio valorativo. Lo mismo ha ocurrido con los verbos deponentes. Utilizadas es-

(10) P. FLOBERT, o.c., p. 110.

(11) P. FLOBERT, o.c., p. 112. 
tas obras para enseñar a los clerici la lengua latina y empleadas por ellos como obras de consulta, es lógico pensar que debería encontrarse una situación similar en los escritos latinos de la época, siempre subordinada al nivel cultural del autor.

Visto cuál era el panorama de los deponentes en ambas obras desde una perspectiva sincrónica, vamos a observarlo diacrónicamente con ayuda del estudio repetidamente mencionado de Flobert.

1.- En primer lugar tenemos un grupo considerablemente numeroso formado por aquellos verbos deponentes que aún lo continúan siendo sin que se les señalen alteraciones ni concurrencias de ningún tipo, aunque muchos de ellos sufrieran en algún momento la competencia de formas activas y pasivas. Son verbos de uso más o menos abundante a lo largo de la latinidad precedente como adulor, aduersor, altercor, arbitror, argumentor, aucupor, auspicor, auxilior, bacchor, blandior, causor, commentor, conor, contemplor, conuicior, conuiuor, cunctor, dignor, dimetior, dominor, emulor, epulor, expergiscor, fabulor, famulor, fateor, defetiscor, for, frumentor, fruor, frustror, fungor, furor, glorior, gradior, grassor, gratificor, grator, gratulor, iaculor, imaginor, imitor, insidior, iocor, irascor, labor, lamentor, latrocinor, letor, liceor, liquor, loquor, lucror, luctor, machinor, medeor, meditor, mentior, mercor, metior, metor, minitor, minor, miror, misereor, miseror, moderor, molior, morior, nanciscor, nascor, negocior, nitor, obliuiscor, operor, opinor, opitulor, ordior, orior, paciscor, patior, peregrinor, periclitor, philosophor, piscor, polliceor, populor, potior, precor, prestolor, proelior, proficiscor, queror, ratiocinor, recordor, reor, rixor, sciscitor, scrutor, sector, sequor, sermocinor, solor, sortior, spacior, speculor, stipulor, stomachor, testificor, testor, tueor, tumultuor, tuor, ulciscor, utor, uagor, uaticinor, uenor, uereor, uescor. También se recogen otros mucho más escasamente atestiguados: antestor, ariolor, arquitector, controuersor, cornicor (creación de Persio tomada por Jerónimo y recogida por escolios, gramáticos y glosistas), lenocinor, licitor, morigeror, ominor, otior, pabulor, patrocinor, ruspor, scitor, scortor, tergiuersor, uerecundor, algunos únicamente atestiguados en los primeros siglos (apiscor, auctionor, cauponor, nugor, pollicitor, uitulor); otros, más esperables, de época tardia (anxior, fistulor, fornicor, meretricor, meridior, peculor, pigritor, principor, pugillor, retrogrador, tedior, uelitor, uerbosor). $Y$ lo que puede parecer más curioso, encontramos también verbos apenas atestiguados anteriormente como ampullor (creación de Horacio), contor (hapax de Plauto conocido por los glosistas), ioculor (hapax de Tito Livio), iuuenor (hapax de Horacio que aparece en los glosarios), nenior (en DOSITH. y los glosarios), nouercor (hapax de Sidonio).

Al lado de todos éstos están aquellos deponentes que en estas dos obras presentan paralelamente una forma activa o neutra, algunos de los cuales ya han sido mencionados.

2.- A continuación tenemos el grupo de los verbos originariamente no deponentes que a su vez pueden ser de dos tipos. Primeramente están los que Flo- 
bert 12 denomina variantes deponentes, puesto que es más frecuente su uso como activos o pasivos que como deponentes. De ellos algo más de la mitad son considerados aquí exclusivamente como deponentes: cachinnor, dissentior, eiulor, fruticor, geniculor, iniurior, lacrimor (antiguamente neutro), mereor (antiguamente activo), naufragor, propicior, remedior, spatulor, uersificor. Con fluctuaciones encontramos: adulteror (neutro o deponente), carminor, delector (activo, neutro o deponente), febricitor (neutro o deponente), iurgor (activo, neutro o deponente), lapsor (a veces, aunque raro, se encuentra como deponente), luxurior (neutro o deponente), nequitor (neutro o deponente), ructor (neutro, activo o deponente), zelor (activo o deponente).

En segundo lugar, están los llamados neo-deponentes ${ }^{13}$, de los que unos son permanentemente deponentes: conuersor, infirmor, stabulor (antiguamente neutro); otros presentan alternancias: amico / amicor (el activo rige acusativo y dativo y significa «amicum facere», el deponente rige dativo y significa «amicum esse»), conflictor, inicior, inimico / inimicor (paralelo a amico/r), memoror.

3.- Pero no se limitan siempre a continuar lo que ya existía en el pasado, también proporcionan algunas novedades. Por una parte hallamos una serie de verbos tradicionalmente activos que han sufrido aquí su transformación en deponentes:

-Augmento: verbo activo, si bien en GLOSS. IV 312, 17: augmentalur crescit. Para el Verbiginale puede encontrarse como activo o deponente. Rige acusativo y significa «ampliare».

-Concordo: «concordem esse uel fieri». Este activo puede hallarse según el Catholicon bajo una doble forma: concordo / concordor.

- Consternor: puesto que ya Prisciano lo menciona como deponente, no es de extrañar que aquí también se le catalogue como tal.

- Crassor: todas las formas atestiguadas de este verbo son pasivas, excepto GLOSS. II 400, $10^{14}$. Para nuestros léxicos puede aparecer como activo o deponente. En el primer caso rige acusativo y significa «crassum facere», en el segundo es absoluto y significa «esse uel fieri crassum».

- Dementor: puede encontrarse como activo con el significado de «furiosum facere» y acusativo, como neutro absoluto significando «furere» y también como deponente con esos mismos significados.

-Episcopor: este verbo, que aparece como activo en inscripciones cristianas, es considerado deponente por estas obras.

-Iantor: «comedere ante prandium, soluere ieiunium». Puede ser neutro o deponente.

-Latinizo: activo para el Catholicon, neutro o deponente para el Verbiginale.

-Latinor: siempre deponente.

(12) o.c., pp. 195 y ss.

(13) Denominación tomada de P. FLOBERT, o.c.

(14) Th. L. L. (s.v.). 
-Muscipulor. siempre deponente, lo mismo que precior con sus compuestos apprecior y deprecior, spiculor, stupro y su compuesto constupror.

-Uaricor. neutro, pero con más frecuencia es deponente. Lo mismo les ocurre a sus compuestos diuaricor y preuaricor.

Junto a esas transformaciones de verbos ya existentes, podemos encontrar también nuevas creaciones del latín medieval:

-Accidior: «tristari, anxiari». Verbo deponente absoluto, derivado del substantivo accidia.

-Aliricor (?): sólo lo testimonia el Catholicon, aunque puede tratarse de una confusión gráfica por altercor. Se trata de un derivado de alter, que puede encontrarse tanto bajo forma activa como deponente, y significa «litigare, certare».

-Archiepiscopor: «esse uel fieri archiepiscopus uel archiepiscopatum habere». Verbo deponente absoluto, derivado de archiepiscopus.

-Aristor: «aristas colligere». Verbo deponente absoluto, derivado de arista.

-Aruspicor: «diuinare». Verbo deponente, se construye con acusativo, deriva de aruspex

- Assensor: «adulari». Verbo deponente derivado de assencio.

-Atticinor: «ad ignem ticiones aduicinare». Deponente compuesto de ad y ticino

-Conuentor-aris: «conuentum facere uel celebrare». Deponente absoluto, derivado de conuentus - us.

-Corniculor: «uocem cornicis imitari uel inutiliter loqui uel garrire». Verbo deponente, derivado de cornix.

-Crissor: «fricare, ceuere». Verbo deponente absoluto o con acusativo. Primitivo.

-Depellicor. «decipere». Verbo deponente construido con acusativo, compuesto de de y pellicio.

-Fluuior. «abundare». Verbo neutro o deponente absoluto, derivado de fluuius. Para ilustrar su utilización como deponente se da la siguiente cita: unde Iosephus in VIII. ${ }^{\circ} X^{\circ}$ : "nil inter eos aut dicior fluuiatur aut pauperior defraudaturn.

-Iantaculor: «comedere ante prandium, ieiunium soluere». Verbo neutro o deponente, construido con acusativo, deriva de ianto.

-Inclunior: verbo activo o deponente. En el primer caso significa «per clunes incuruare uel eleuare», en el segundo «concubitum pati». Compuesto de in y clunis.

-Inclunitor: «per clunes incuruare uel eleuare». Verbo activo o deponente, transitivo, derivado del anterior.

-Inficior: «contra ueritatem mendatio uti, rem debitam negari cum a creditore deposcitun. Verbo deponente, compuesto de in y facio, se construye con acusativo.

-Ingenior: «ingenium exercere, ingeniose se habere». Verbo deponente, 
derivado de ingenium. Cuando aparece con el significado de «decipere», rige acusativo.

-Mechor. «adulterari». Verbo deponente absoluto o con acusativo, primitivo. Lo mismo se puede decir de su compuesto commechor.

-Miniculor: «auxiliari». Verbo deponente, construido con dativo, derivado de miniculum.

-Nictitor. «frequente nocte uigilare». Verbo neutro o deponente, frecuentativo de nictor. Sólo aparece en el Catholicon.

-Petulor. «importune petere et proprie cohitum luxuriari, lasciuie animum dare». Verbo deponente absoluto, aunque a veces lo puede acompañar un acusativo. Deriva de petulus.

-Pontificor: «pontificatum regere uel habere uel more pontificis se habere». Verbo deponente absoluto, derivado de pontifex.

-Prouerbior. «prouerbia dicere uel prouerbiis uituperare». Verbo deponente transitivo, derivado de prouerbium.

-Racionor: «racionabiliter loqui uel tractare». Verbo deponente absoluto, derivado de ratio. Sólo lo testimonia el Verbiginale.

-Suffraginor: «flectere genua». Verbo deponente absoluto, derivado de suffragines.

-Testamentor. «testamentum facere». Verbo deponente transitivo, derivado de testamentum.

-Titionor: «ticiones preparare, eos in ignem mittere». Verbo deponente transitivo derivado de titio.

-Transalpinor: «ultra Alpes ire». Verbo deponente absoluto, derivado de transalpinus $-a-u m$.

El número de nuevas creaciones viene a suponer aproximadamente un $7 \%$ dentro de los verbos considerados deponentes (sin contar los compuestos mediante preverbios que proliferan por doquier). Su mera presencia es suficiente para mostrar que dentro de una lengua que aumentó el caudal léxico heredado, el grupo de los deponentes es todavía una clase productiva y, por lo tanto, viva también dentro del latín medieval, a la que se sienten pertenecer particularmente los verbos derivativos o los nuevos compuestos o derivados de los antiguos deponentes.

Si hay verbos activos considerados deponentes, también se presenta el fenómeno contrario, deponentes de cuya naturaleza como tales no queda el menor rastro: alapo, alumpno, arguto, caluo, capillo, carino, chirographo, fauello, martirizo, modifico, palo, radico, rumino, secundo, etc.

En resumen, hemos visto cómo Verbiginale y Catholicon presentan un sistema verbal en el que se conserva la categoría de los verbos deponentes, que, después de todo, son una de las más llamativas peculiaridades de la lengua latina, en concreto del verbo, y de las que, además, la diferencian de las ya existentes lenguas romances. $Y$ la conservan con una moderación que es digna de mencionarse: no hacen proliferar el número de estos verbos en exceso hasta extremos que los convertirían en meros artificios manieristas. Sigue presente el movimiento interno que existió desde siempre dentro de esta clase 
de verbos y tiene las mismas tendencias que en el pasado: hay verbos activos considerados como deponentes, verbos deponentes considerados como activos o neutros y otros que pueden adoptar este u otro género. Normalmente la concurrencia de las formas deponentes junto con otras no tiene ninguna justificación, la mayoría de la veces se trata de meras variantes morfológicas intercambiables en cualquier contexto. Sin embargo, no se puede observar una presencia o un aumento excesivo de variantes deponentes o neo-deponentes. Si a esta situación descrita le añadimos la existencia de un cierto número de nuevas creaciones (aparte de nuevas composiciones o derivaciones sobre bases ya deponentes), podemos concluir que el latín medieval conserva aún con alguna vida esta categoría verbal y con un aspecto no muy diferente al de épocas pasadas. 
